\title{
Breast implant-associated anaplastic large cell lymphoma: a case report with a history of spontaneously resolved late seroma
}

Do Yeon Kim ${ }^{1}$, Joon Hur ${ }^{2}$, Woo Yeon $\mathrm{Han}^{2}$, Kyunghyun Min ${ }^{3}$, Jong Won Lee ${ }^{4}$, Jin Sup Eom ${ }^{2}$, Hyun Ho Han ${ }^{2}$, Eun Key Kim²

${ }^{1}$ The WOORI Plastic Surgery Clinic, Seoul; ${ }^{2}$ Department of Plastic Surgery, Asan Medical Center, University of Ulsan College of Medicine, Seoul; ${ }^{3}$ Department of Plastic and Reconstructive Surgery, Hanyang University College of Medicine, Seoul; ${ }^{4}$ Department of Surgery, Asan Medical Center, University of Ulsan College of Medicine, Seoul, Korea

We appreciate valuable discussions with Professor Il-Kug Kim, Department of Plastic Surgery, Yeungnam University College of Medicine, Daegu, Korea. We also express our gratitude to Dr. Jongwon Lee, Department of Pathology, Asan Medical Center, University of Ulsan College of Medicine, Seoul, Korea.
We report a case of breast implant-associated anaplastic large cell lymphoma (BIA-AL$\mathrm{CL}$ ), which had a history of spontaneous resorption of late seroma before diagnosis. A 47-year-old woman with a history of augmentation mammoplasty with round textured implants in January 2013 presented with a swelling on her right breast 6 years later, which was diagnosed as late seroma with suspected intracapsular rupture using ultrasonography (USG). Although aspiration was not done at the time of the initial USG, the seroma resolved spontaneously within weeks. A further workup proceeded with USGguided aspiration followed by magnetic resonance imaging. Cytology of the aspirated fluid showed atypical cells. Cell block cytology and immunohistochemical staining confirmed the diagnosis of BIA-ALCL. En bloc resection with total capsulectomy and explantation was performed as curative surgery. Pathologic stage pT2NoMo was confirmed and the patient was followed up without further treatment. Although the classic presentation of BIA-ALCL is known as late persistent seroma, an atypical manifestation such as spontaneous resorption may occur, as in the current case. A high level of suspicion and a thorough investigation with appropriate modalities will make it possible to detect this rare and potentially devastating disease.

Keywords Breast implants / Lymphoma / Seroma

\section{INTRODUCTION}

Breast implant-associated anaplastic large cell lymphoma (BIAALCL) is a rare and highly curable subtype of non-Hodgkin lymphoma [1]. It is most frequently associated with the insertion of textured breast implants [2]. The lifetime risk of BIA-ALCL is currently estimated to be 1:2,207-1:86,029 for various types of tex-

Received: Jun 24, 2021 Revised: Jul 6, 2021 Accepted: Jul 9, 2021

Correspondence: Eun Key Kim Department of Plastic Surgery, Asan Medical Center, University of Ulsan College of Medicine, 88 Olympic-ro 43gil, Songpa-gu, Seoul 05505, Korea

Tel: +82-2-3010-3600, Fax: +82-2-476-7471, E-mail: nicekek@korea.com

Copyright @ 2021 The Korean Society for Aesthetic Plastic Surgery.

This is an Open Access article distributed under the terms of the Creative Commons Attribution Non-Commercial License (https://creativecommons.org/licenses/by-nc/4.0/) which permits unrestricted non-commercial use, distribution, and reproduction in any medium, provided the original work is properly cited. www.e-aaps.org tured implants from different manufacturers [3]. An association between cutaneous T-cell lymphoma and silicone breast implants was first mentioned in 1995, with three cases confirmed [4]. The first patient diagnosed with BIA-ALCL was reported in 1997, and as of April 24, 2020, 322 suspected/confirmed cases in the United States and 903 cases worldwide have been reported [5]. The Food and Drug Administration identified a possible association between breast implants and BIA-ALCL development in 2011. In 2016, the World Health Organization designated BIA-ALCL a distinct entity from T-cell lymphoma [6]. The typical symptoms of BIA-ALCL are reported to be persistent swelling, pain, and formation of a mass. Seroma formation around the breast implant is often observed. Patients who present with effusion or seroma (70\%) and/or distinct mass formations (30\%), as well as patients with breast masses, showed worse overall survival and progression-free survival [7]. Immediate surgery for implant removal and capsulectomy is required as curative treatment [8]. Otherwise, the lymphoma can invade outside 
the fibrous capsule and lead to death.

Late-onset persistent seroma around breast implant represents the classic clinical presentation in up to $80 \%$ of women with BIAALCL, and the risk of BIA-ALCL in women who present with delayed seromas is up to $10 \%$ [9]. With increasing awareness of this disease, a few case reports have been published in Asia [10,11], including the current case from the pathologist's point of view [12]. This Korean female patient was presented with delayed swelling of her right breast 81 months after implantation and was diagnosed with late seroma on initial ultrasonography (USG). Although the seroma disappeared spontaneously in a few weeks, a further workup confirmed the diagnosis of BIA-ALCL. This case report focuses on the clinical pitfalls in the diagnostic process of an uncommon presentation of BIA-ALCL.

Our institutional review board granted exempt status for this protocol since this is a single case report presenting a factual description of the clinical features and outcomes without any additional testing, evaluation, analysis, or review of others for comparison.

\section{CASE REPORT}

A 47-year-old woman presented with a history of bilateral augmentation mammoplasty in January 2013, with the insertion of Allergan Natrelle silicone-filled textured breast implants (Style 115, BIOCELL Textured Round Midrange Projection Gel Filled Breast Implant, $253 \mathrm{cc}$ ). She experienced swelling on the right breast, which became enlarged and tense in October 2019 (81 months after implantation). Pain and skin color changes were absent. She visited another center where USG of her breasts was done. Intracapsular rupture with seroma formation was suspected (Fig. 1). Although percutaneous needle aspiration was not done at the time, the swell-

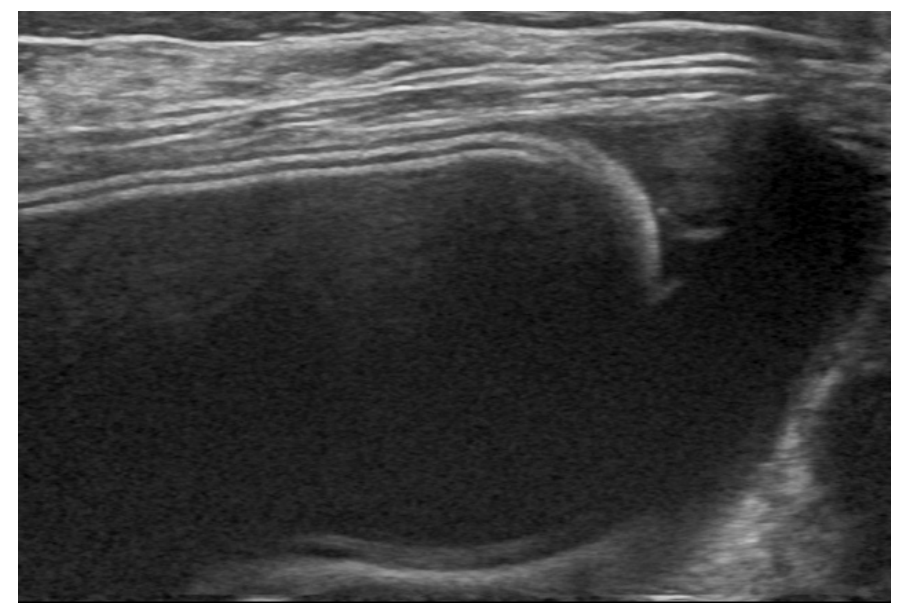

Fig. 1. Initial ultrasonography (USG) findings. A patient with late swelling of the right breast showed fluid collection with an inner capsule discontinuity on initial USG, diagnosed as late seroma with suspected intracapsular rupture. ing resolved spontaneously within weeks of follow-up.

The patient presented to us on November 27, 2019 for further evaluation and implant removal. There was no apparent asymmetry on gross observation (Fig. 2). In light of the patient's history of delayed-onset seroma, aspiration for cytology and magnetic resonance imaging (MRI) of the breasts were performed due to suspicion of BIA-ALCL. USG showed fluid collection with capsule discontinuity, suggesting a ruptured implant on the right side; however, this possibility was proven false later upon surgical exploration. The collected fluid was scant for aspiration cytology with less than $10 \mathrm{~mL}$ of the seroma collected. The aspirated sample included atypical lymphocytes with irregular-shaped nuclei, displaying kidneyshaped nuclei and binucleation, which raised suspicion as hallmark cells of BIA-ALCL. On MRI, a collection of fluid on the posterior

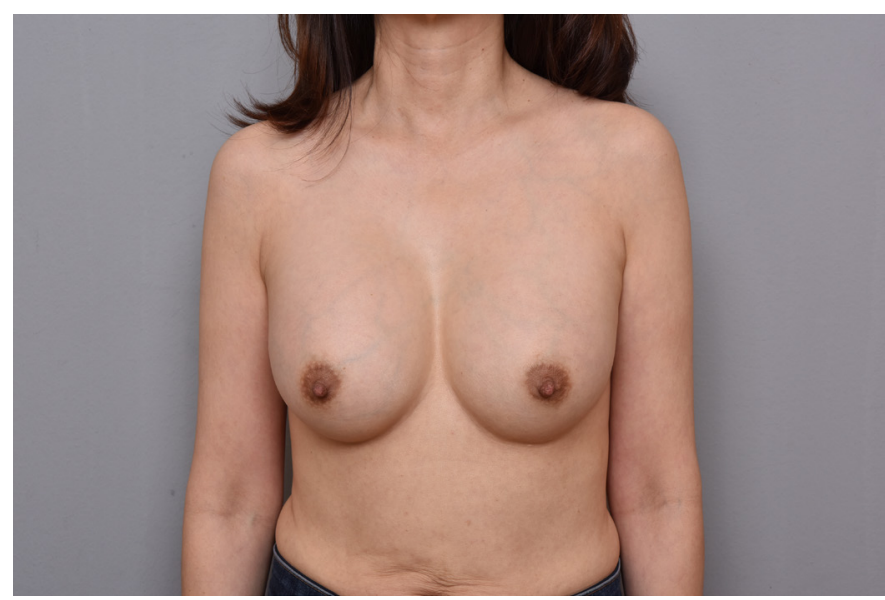

Fig. 2. Preoperative clinical photograph. Preoperatively, there was no apparent asymmetry on gross observation.

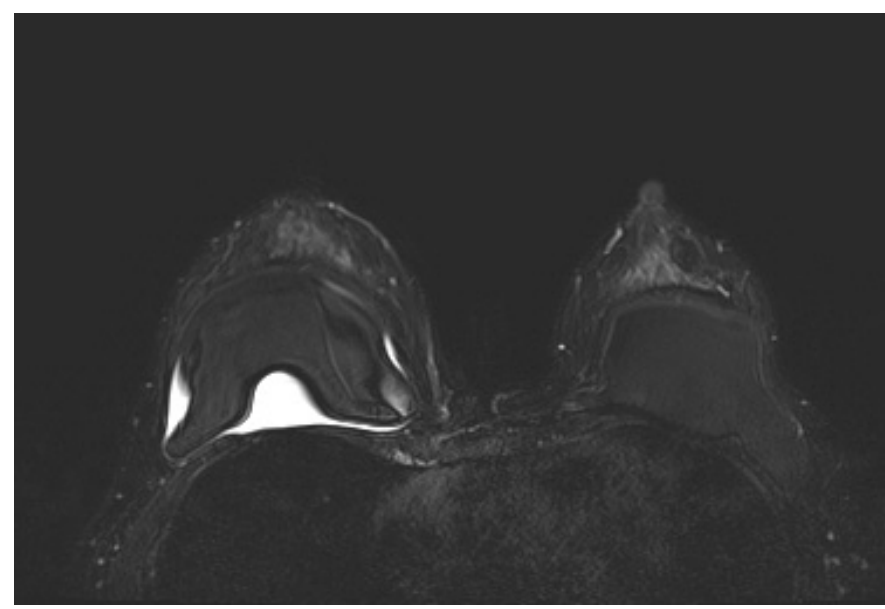

Fig. 3. Preoperative magnetic resonance imaging findings. Fluid collection mainly posterior to the right breast implant was observed, and the intact implant was covered with a thickened and enhanced capsule. No mass-like lesion was observed. 

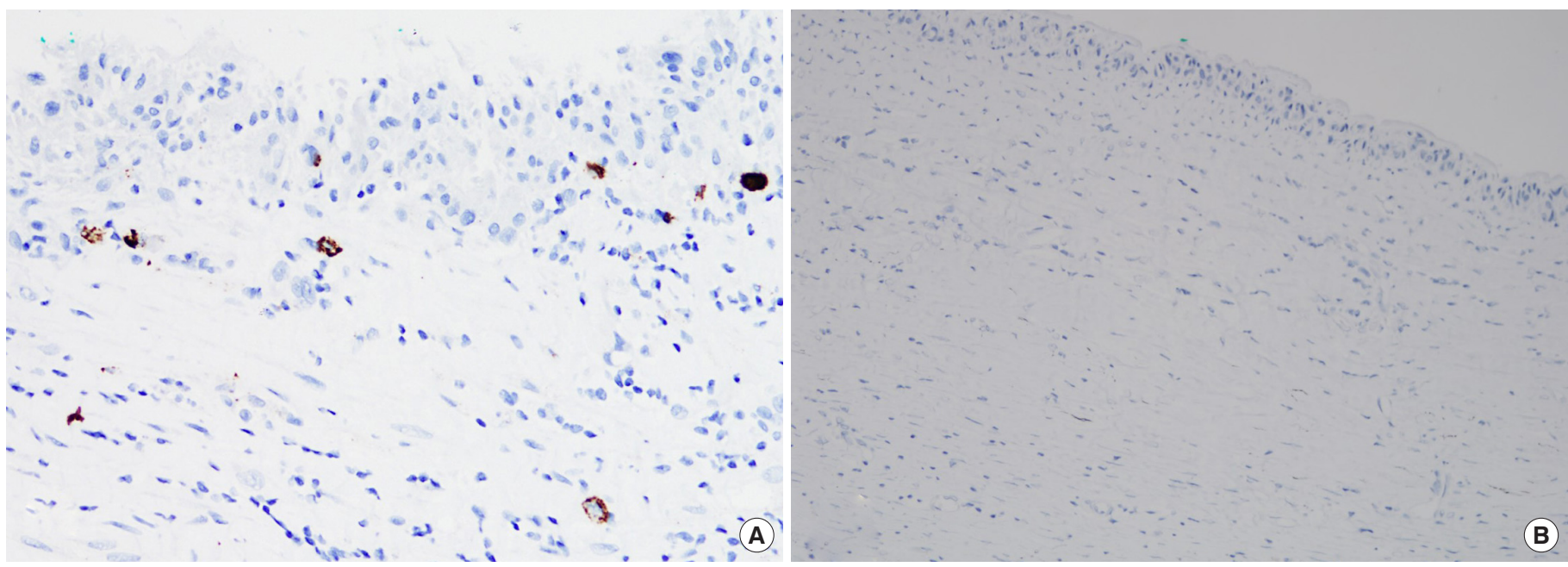

Fig. 4. Immunohistochemistry of the surgical specimen (capsule). (A) Large atypical cells showed diffuse CD30 immunopositivity ( $\times 100$ ). (B) The cells were confirmed as negative for anaplastic lymphoma kinase (ALK) ( × 100).

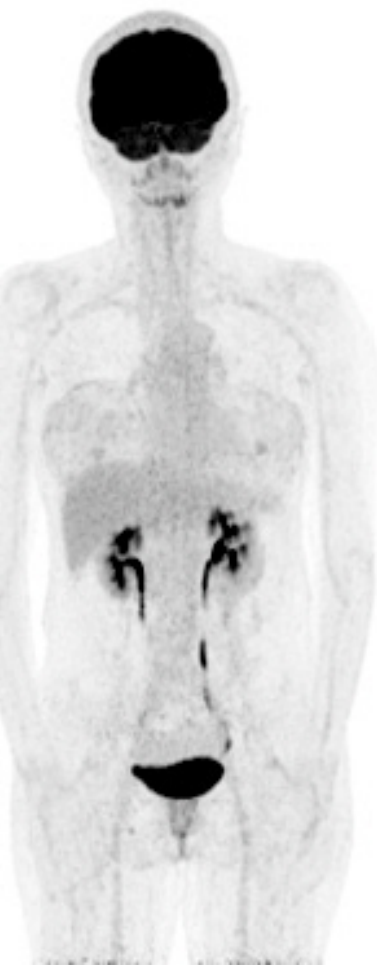

Fig. 5. Preoperative positron emission tomography-computed tomography (PET-CT) scan. Whole-body PET-CT scan revealed only faint capsular uptake with a peri-implant fluid collection in the right breast, and no specific nodular uptake was observed.

side of the right breast was observed (Fig. 3), which would have been missed on the previous USG, with the intact breast implant covered with a thickened and enhanced fibrous capsule. No masslike lesion was observed on MRI or computed tomography (CT). A multidisciplinary breast conference was held and a second-look,
USG-guided percutaneous needle aspiration was recommended for cell block immunohistochemistry (IHC). Large atypical T cells with irregular nuclei positive for $\mathrm{CD} 30$ and $\mathrm{CD} 3$ and negative for anaplastic lymphoma kinase were observed; these findings were suggestive of BIA-ALCL (Fig. 4). The patient underwent a staging workup with whole-body positron emission tomography (PET), revealing faint capsular uptake with a peri-implant fluid collection in her right breast and no specific nodular uptake (Fig. 5). CT scans of the neck, chest, abdomen, and pelvis showed non-specific findings, while blood tests and bone marrow biopsy were also negative for lymphoma cells.

In the operating room, implant removal with total capsulectomy in the left breast and en bloc resection in the right breast, including partial excision of the pectoralis major muscle, were performed (Fig. 6). Sentinel lymph node biopsy revealed no involvement of the lymph nodes; therefore, lymph node dissection was not indicated. A gross specimen showed a thickened fibrous capsule with no definite mass-like lesion, with a few plaque-like lesions (Fig. 7). Despite our request, the pathology department did not identify specific lesions of nodularity that suggested lymphoma. The inner side of the capsule demonstrated a multifocal yellowish plaque-like material and detachment from the implant (seen as glossy surface areas), which was suggestive of previous seroma formation, without any sign of rupture of either the implant or the capsule. In the histologic sections analyzed by the pathology department, infiltrations of large atypical lymphocytes positive for CD30 and negative for anaplastic lymphoma kinase were observed throughout the inner surface of the fibrous capsule on the right side, with pathologic stage T2 (Ann-Arbor stage 1EA). The patient was discharged with no acute complications on postoperative day 3 . Three weeks after the operation, she visited the multidisciplinary outpatient department and follow-up was recommended every 6 months with USG, 

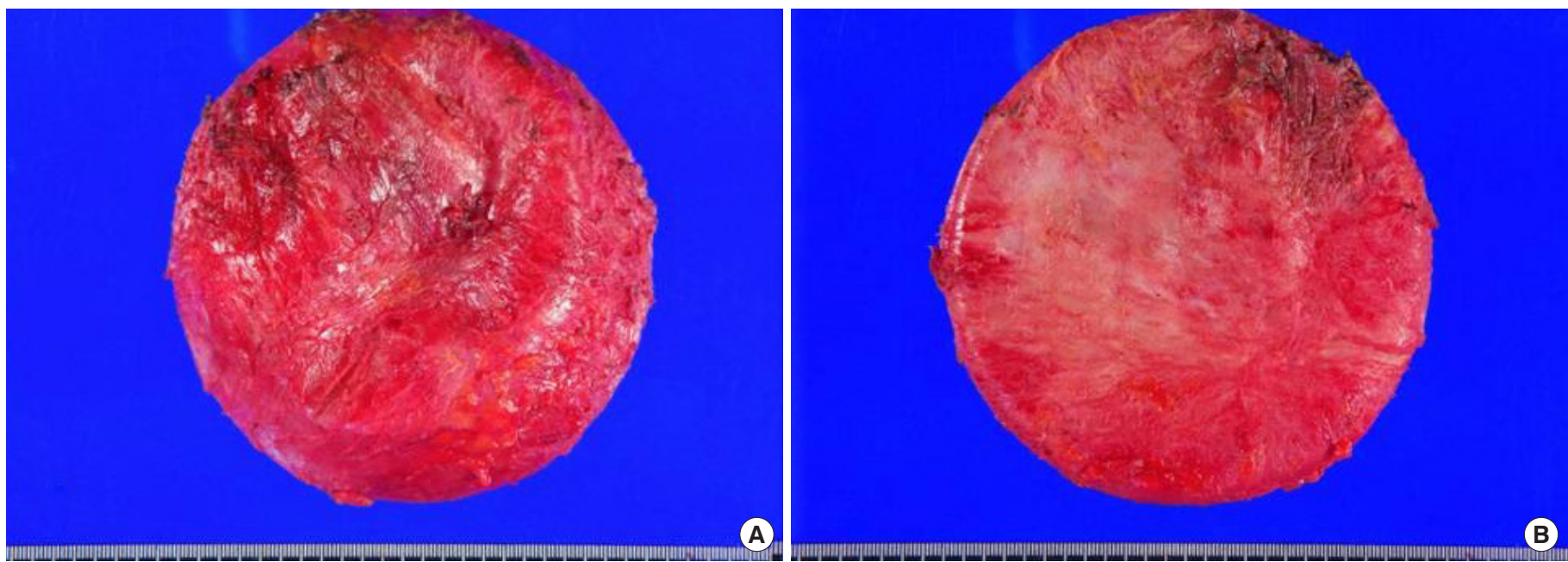

Fig. 6. En bloc resection with total capsulectomy and explantation was performed, including partial excision of the pectoralis major muscle. Anterior (A) and posterior (B) gross view.

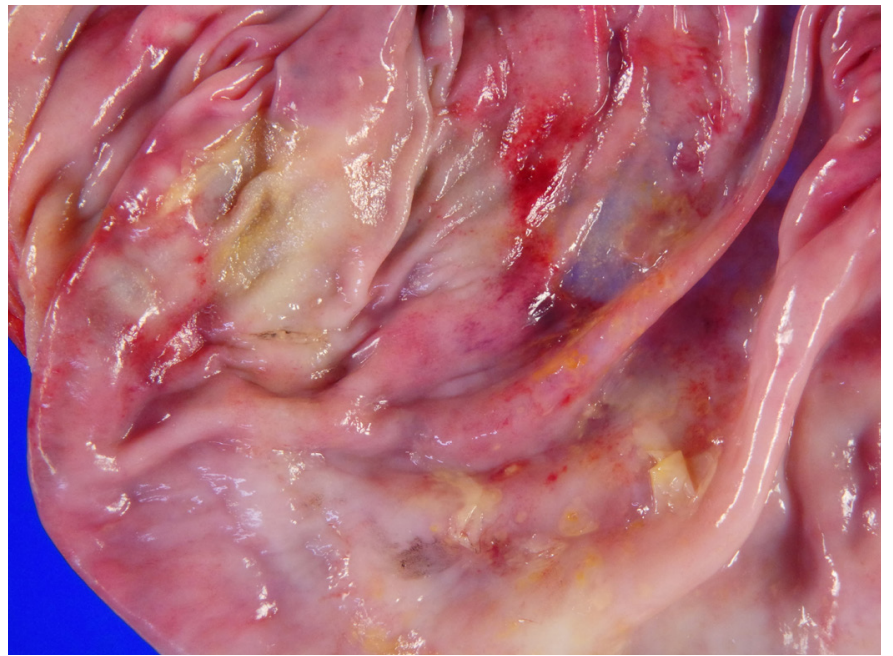

Fig. 7. Inner side of the capsule. A gross specimen showed a thickened fibrous capsule with no definite mass-like lesion, a few plaquelike lesions, and partial detachment from the implant seen as glossy inner surface areas.

MRI, and blood tests without further treatment. Of note, the nuclear medicine department concluded that whole-body PET showed completely different results from typical cases of lymphoma, which show marked hypermetabolic activity, making postoperative surveillance difficult. The patient has been followed up by a multidisciplinary team for breast parenchymal lesions, and there has been no evidence of disease recurrence.

\section{DISCUSSION}

Clinicians' concerns regarding BIA-ALCL have increased during the last couple of decades, and a few cases have been reported in
Asians. Considering the clinical manifestation that has been reported worldwide, we believe that this case requires clinical attention, as it initially showed a typical clinical presentation of delayedonset seroma but then spontaneously resolved with no other specific symptoms or signs, making it difficult to suspect BIA-ALCL in a straightforward manner.

The common clinical features of BIA-ALCL include persistent swelling due to fluid collection between the breast implant and fibrous capsule, pain, and the presence of a mass; these findings are observed some years after implant placement (range, 1-32 years; median, 9 years) $[8,9]$. Except for the time to presentation, the clinical presentation of our case did not coincide with those features. Even the initial USG did not suggest a diagnosis of BIA-ALCL; instead, it gave the impression of effusion with implant rupture, which proved to be false upon a further workup and surgery. Although USG is generally recommended as the first-line screening test, a small seroma, especially when located at the posterior side of the implant, may be misdiagnosed, and MRI is recommended if there is clinical suspicion.

Another point related to the pathologic review is that gross inspection of the involved capsule did not allow us to distinguish specific sites of lymphoma involvement. Although positive results could be obtained on histologic sectional IHC, the pathology department could not identify any particular lesions in the specimen. This suggests that when a patient presents with symptoms that do not clearly indicate a BIA-ALCL diagnosis, and even if the intraoperative findings do not seem to be relevant, suspicion should be maintained and pathologic studies should follow.

The 2019 National Comprehensive Cancer Network consensus guidelines suggest a standardized approach to the diagnosis and management of BIA-ALCL. If a patient presents with symptoms of effusion, mass, and a skin rash/ulcer more than a year after implan- 
tation, USG or MRI should be done. Over $50 \mathrm{~mL}$ of fine-needle aspiration fluid for effusion or incisional/core needle biopsy of a mass is required for cytologay, pathology, cell block-flow cytometry, and CD30 IHC. If a diagnosis of lymphoma remains indeterminate, clinicians should refer a patient to a tertiary cancer center for a second pathologic consultation.

When a diagnosis of BIA-ALCL is confirmed, a report to the PROFILE registry (Patient Registry and Outcomes for Breast Implants and Anaplastic Large Cell Lymphoma Etiology and Epidemiology registry; https://www.thepsf.org/research/registries/profile) [13] should be made and a multidisciplinary discussion is recommended among plastic surgeons, oncologists, surgical oncologists, and pathologists. The preoperative lymphoma workup and staging should be followed with blood tests and a PET/CT scan. En bloc resection, including total capsulectomy, explantation, and excisional biopsy of the mass and lymph nodes, is recommended for localized disease (IA-IC, IIA), followed by postoperative surveillance with follow-up visits every 3-6 months for 2 years with CT or PET/CT every 6 months for 2 years when indicated. In cases of advanced disease (stage IIB-IV) or incomplete excision, systemic therapy and radiotherapy are recommended [14].

In our case, faint capsular uptake around the breast implants was observed vaguely without any nodular uptake on PET in the preoperative workup. This finding seems plausible, considering the fact that the majority (70\%) of reported BIA-ALCL cases were observed to show only effusion around the breast implant, without the formation of any solid mass. This implies that BIA-ALCL shows a different course of progression when compared to typical solid tumors. Thus, meticulous observations with multiple recommended modalities should be emphasized during postoperative surveillance for the detection of any abnormal findings.

We found that our case was difficult to clinically distinguish from other cases of benign late seromas, which are relatively common. The diagnosis of BIA-ALCL was missed on the first visit in this case. Even after curative surgical treatment at our center, the course of the disease if its clinical manifestations had been neglected and left untreated remains unclear. We do not know whether spontaneous regression of the seroma in the current case was associated with regression of BIA-ALCL, or a waxing-and-waning course of the disease. In 2018, two cases of spontaneous regression and resolution of the disease were reported in Australia [15]. Although both cases were treated with explantation and capsulectomy, it seems that BIA-ALCL can be self-limiting, resulting in subclinical or negligible cases that are not detected by clinicians or even by the patients themselves. Currently, there is neither sufficient evidence nor a strong consensus on the treatment strategy for this group of patients or the final results of such a clinical course. Thus, further investigation is required.

\section{NOTES}

\section{Conflict of interest}

No potential conflict of interest relevant to this article was reported.

\section{Ethical approval}

The study was exempted from approval by the Institutional Review Board of Asan Medical Center and performed in accordance with the principles of the Declaration of Helsinki.

\section{Patient consent}

The patient provided written informed consent for the publication and the use of her images.

\section{ORCID}

Do Yeon Kim

Joon Hur

Woo Yeon Han

Kyunghyun Min

Jong Won Lee

Jin Sup Eom

Hyun Ho Han

Eun Key Kim

\section{REFERENCES}

1. Brody GS, Deapen D, Taylor CR, et al. Anaplastic large cell lymphoma occurring in women with breast implants: analysis of 173 cases. Plast Reconstr Surg 2015;135:695-705.

2. Doren EL, Miranda RN, Selber JC, et al. U.S. epidemiology of breast implant-associated anaplastic large cell lymphoma. Plast Reconstr Surg 2017;139:1042-50.

3. American Society of Plastic Surgeons. Breast implant-associated anaplastic large cell lymphoma (BIA-ALCL) [Internet]. Arlington Heights, IL: American Society of Plastic Surgeons [cited 2020 Jun 20]. Available from: https://www.plasticsurgery.org/patient-safety/breast-implantsafety/bia-alcl-summary.

4. Duvic M, Moore D, Menter A, et al. Cutaneous T-cell lymphoma in association with silicone breast implants. J Am Acad Dermatol 1995; 32:939-42.

5. American Society of Plastic Surgeons. BIA-ALCL physician resources [Internet]. Arlington Heights, IL: American Society of Plastic Surgeons [cited 2020 Jun 20]. Available from: https://www.plasticsurgery.org/ for-medical-professionals/health-policy/bia-alcl-physician-resources.

6. Swerdlow SH, Campo E, Pileri SA, et al. The 2016 revision of the World Health Organization classification of lymphoid neoplasms. Blood 2016; 127:2375-90.

7. Neligan PC, Nahabedian MY. Plastic surgery. Volume 5. 4th ed. London: Elsevier Saunders; 2018.

8. Quesada AE, Medeiros LJ, Clemens MW, et al. Breast implant-associ- 
ated anaplastic large cell lymphoma: a review. Mod Pathol 2019;32:16688.

9. Marra A, Viale G, Pileri SA, et al. Breast implant-associated anaplastic large cell lymphoma: a comprehensive review. Cancer Treat Rev 2020; 84:101963.

10. Kim IK, Hong KY, Lee CK, et al. Analysis of the molecular signature of breast implant-associated anaplastic large cell lymphoma in an Asian patient. Aesthet Surg J 2021;41:NP214-22.

11. Thienpaitoon P, Disphanurat W, Warnnissorn N. Breast implant-associated anaplastic large cell lymphoma in an Asian patient: the first case report from Thailand. Arch Plast Surg 2020;47:478-82.

12. Lee J, Cho $\mathrm{H}$, Yoon $\mathrm{DH}$, et al. Breast implant-associated anaplastic large cell lymphoma: the first South Korean case. J Pathol Transl Med
2020;54:432-4.

13. The Plastic Surgery Foundation. Profile [Internet]. Arlington Heights, IL: The Plastic Surgery Foundation [cited 2020 Jul 12]. Available from: https://www.thepsf.org/research/registries/profile.

14. Clemens MW, Jacobsen ED, Horwitz SM. 2019 NCCN Consensus Guidelines on the diagnosis and treatment of breast implant-associated anaplastic large cell lymphoma (BIA-ALCL). Aesthet Surg J 2019; 39(Suppl_1):S3-13.

15. Fleming D, Stone J, Tansley P. Spontaneous regression and resolution of breast implant-associated anaplastic large cell lymphoma: implications for research, diagnosis and clinical management. Aesthetic Plast Surg 2018;42:672-8. 